Article

\title{
An Event-Based Resource Management Framework for Distributed Decision-Making in Decentralized Virtual Power Plants
}

\author{
Jianchao Zhang, Boon-Chong Seet * and Tek Tjing Lie \\ Department of Electrical and Electronic Engineering, Auckland University of Technology, Auckland 1010, \\ New Zealand; jizhang@aut.ac.nz (J.Z.); tlie@aut.ac.nz (T.T.L.) \\ * Correspondence: bseet@aut.ac.nz; Tel.: +64-09-921-9999
}

Academic Editor: Peter V. Schaeffer

Received: 5 May 2016; Accepted: 22 July 2016; Published: 28 July 2016

\begin{abstract}
The Smart Grid incorporates advanced information and communication technologies (ICTs) in power systems, and is characterized by high penetration of distributed energy resources (DERs). Whether it is the nation-wide power grid or a single residential building, the energy management involves different types of resources that often depend on and influence each other. The concept of virtual power plant (VPP) has been proposed to represent the aggregation of energy resources in the electricity market, and distributed decision-making (DDM) plays a vital role in VPP due to its complex nature. This paper proposes a framework for managing different resource types of relevance to energy management for decentralized VPP. The framework views VPP as a hierarchical structure and abstracts energy consumption/generation as contractual resources, i.e., contractual offerings to curtail load/supply energy, from third party VPP participants for DDM. The proposed resource models, event-based approach to decision making, multi-agent system and ontology implementation of the framework are presented in detail. The effectiveness of the proposed framework is then demonstrated through an application to a simulated campus VPP with real building energy data.
\end{abstract}

Keywords: resource management framework; distributed decision-making; virtual power plant; smart grid

\section{Introduction}

The Smart Grid refers to the next-generation electrical power grid, which aims to achieve smarter energy usage and integration of distributed energy resources (DERs) by harnessing advanced Information and Communication Technologies (ICTs) to provide an intelligent bi-directional network for both electricity and information delivery. In the Smart Grid, a large number of different devices, such as intelligent electronic devices (IEDs), sensors, actuators, and energy sources distributed throughout the electricity system will be interconnected and communicating over an information network for metering, monitoring, control and protection. Hence, Smart Grid can be considered as a large-scale, heterogeneous and distributed cyber physical system (CPS) [1].

In the literature, Smart Grid is also sometimes referred to as the Internet of Energy, and it enables electricity consumers to become active players in the power system by feeding customer-side generated energy back into the main grid or participating in demand response programs. However, the high penetration of customer-side DERs also leads to new problems such as voltage rising with increasing distance from substation and revenue optimization when offering excess DER energy in the energy market [2].

The virtual power plant (VPP) is a concept for aggregating DERs and controllable loads, and presenting them to the main grid as a single energy trader [3]. The VPP can be centralized or 
decentralized, depending on whether the energy planning/scheduling is performed by a central controller, or in a distributed manner by "smart" energy resources.

Herein, the energy planning is referred to as consumption adjustment at some future time (e.g., a day ahead) based on forecast data, and energy scheduling as consumption adjustment in real time or very short notice. Compared to centralized VPP, the decentralized VPP has higher scalability and openness because the VPP operator does not directly control the energy consumption/generation or VPP membership, but only dispatches information such as price signals and requires minimal knowledge about the aggregated resources [4]. The energy planning/scheduling in decentralized VPP could be posed as a distributed decision-making (DDM) problem [5].

The resource management problem in VPP involves different resources from different domains, e.g., power system domain and ICT domain, requiring complex provisioning and planning by collaborating between different resource management systems that are often owned by different customers. This demands a resource management approach that is more agile and flexible than existing ones, and which explicitly considers the interactions between systems in different domains.

This paper focuses on addressing the resource management issues in decentralized VPP and presents resource models, concepts of events and corresponding event processing, based on which an event-based resource management framework is proposed to support distributed decision making in the VPP. As will be further discussed in Section 4, an event in VPP refers to a state transition of, or an action task initiated by a resource. By event-based resource management framework, we refer to a conceptual structure designed to support resource management by providing views on how resources are interrelated and how operations on the resources can be orchestrated based on the transmission and processing of events.

By viewing VPP as a hierarchical structure and abstracting energy consumption/generation as contractual resources, i.e., contractual offerings to curtail load/supply energy, from third party VPP participants, the proposed framework offers flexibility and adaptability to cross-domain system designs, which in turn accelerates the development of energy management applications.

The rest of the paper is organized as follows: Section 2 examines the related standards and works in literature. Section 3 presents the proposed resource management framework and corresponding resource model. Section 4 describes the events and event processing of the proposed framework for distributed decision making. The ontology implementation and simulation analysis of the proposed framework are discussed in Sections 5 and 6, respectively. Finally, Section 7 concludes the paper with some suggestions for future work.

\section{Related Standards and Works}

\subsection{IEC 61850 and IEC $61968 / 61970$}

The International Electrotechnical Commission (IEC) Technical Committee 57 (TC57) [6] provides a reference architecture for electric power systems. The main data semantics and system models are standardized by the IEC 61850 and IEC 61970/61968 suites. Data semantics provided by IEC 61850 are closely related to the functionality of devices in the subsystems of the power grid operator, such as substations, wind power plants, hydro power plants, and DERs [6].

IEC 61970 was developed for exchanging information about the electrical grid and application programming interfaces (API) for energy management systems commonly used in control centers, while IEC 61968 further describes the details of the distribution system in IEC 61970 [7]. Collectively, the IEC 61970/61968 standards constitute part of the IEC Common Information Model (CIM) for modeling the power system semantics [8].

The IEC modeling primarily focuses on the power grid operator's network, and does not encompass other information (e.g., building spatial information) necessary for modeling resources in buildings that can be also active Smart Grid participants. Furthermore, since CIM and IEC 61850 have been separately developed by different workgroups, any mismatches between the models may hinder interoperability between the devices, systems, and applications [9]. 


\subsection{Resource Modeling in Cloud Computing and Internet of Things (IoT)}

Information modeling is an essential process for resource management in Cloud Computing and the Internet of Things (IoT). The Semantic Sensor Network (SSN) Ontology [10] is one of the most widely used information modeling techniques for describing sensors and sensor observations in IoT. The SSN is derived from Descriptive Ontology for Linguistic and Cognitive Engineering (DOLCE) [11] and is based on Ontology Web Language (OWL). In SSN, events are classified into two types: action and process. However, such classification is too general to be usefully applicable to complex CPS such as Smart Grid. Furthermore, SSN lacks domain knowledge, e.g., power system and spatial information, and thus often requires to be used with other ontology modeling techniques.

In cloud computing, resources have also been modeled using application-specific domain knowledge. In [12], the authors proposed multi-dimensional resource modeling for the allocation scheme in cloud computing, in which application-specific knowledge such as processing, computation and communication requirements of the client is considered in order to meet the service level agreements (SLA). In [13], the authors incorporated a user model into their ICT resource framework for storage allocation, modeling their ICT resources based on application-specific domain knowledge of both user and hardware information. However, the above resource modeling focuses on ICT domain and lacks knowledge in other domains that are required by the Smart Grid.

\subsection{Resource Modeling and Distributed Decision Making in Smart Grid}

In recent years, resource information modeling has also attracted much attention from Smart Grid researchers. In [14], the authors proposed an ontology for microgrid management, which was said to address the interoperability between different energy management systems within the microgrid, and the need to model all aspects of energy for multiple management objectives such as reliability and balancing in microgrids. In [15], an ontology for modeling prosumer details down to appliance level was proposed with microgrid events classified into appliance, weather, storage and generator events.

Researchers have also applied the concept of agents and utilized Multi-Agent System (MAS) to implement distributed decision-making (DDM) for energy systems. In [16], the authors defined agents responsible for load, generation and storage management, and proposed an agent profit maximization model, which was applied to an electric vehicle management system based on IEEE 37-bus distribution grid. In [17] an architecture for provisioning of demand response services from aggregated small residential consumers was presented, along with its MAS implementation. A MAS-oriented model for VPP was proposed in [18], in which generation/consumption control was achieved by implementing roles and tasks for producers/consumers whose behaviors are defined by specific use cases. Energy demand forecast was also introduced by integrating artificial neural networks into each agent. However, real time scheduling was not investigated, and the relationships between resources from different domains were not discussed.

In summary, the existing Smart Grid resource modeling techniques have mainly focused on covering all useful information but they lack the capability for resource abstraction that is crucial to VPPs, which are aggregating third party energy resources. Furthermore, existing works such as $[15,18]$ do not model the hierarchical structure of VPP and lack event processing logic that captures the cooperation between VPP components. For a VPP that aggregates multiple buildings, it is not always possible to obtain every detail at the customer site and exert direct control on their consumption/generation patterns. Therefore, resources in such VPPs should be managed with minimum intervention and effort required from VPP operator, while achieving revenue optimization objectives. Moreover, existing MAS approaches mostly focus on market bidding/offering strategies or appliance control, with few or none considering the interoperability between VPP components. 


\section{Resource Management Framework and Corresponding Resource Model}

\subsection{Resource Classification and Resource Management Framework}

In the field of Smart Grids, there is not yet a clear definition of the term "resource management". It is sometimes even confusing to mention the term "resource" because it could refer to the energy offering in the market, equipment in the power system, or the hardware and software in the IT infrastructure.

For example, in IEC 61970 and 61968, two different classes co-exist to represent resources in the power grid: PowerSystemResource for equipment in the electric network, such as a generation unit, and Asset for all other devices owned by the power grid company, such as communication media $[7,8]$.

On the other hand, in cloud computing and IoT, the term "resource" often refers to "a reusable entity that is employed to fulfil a job or request", e.g., processors to perform computation, or objects that can provide data [19] such as sensors.

This paper proposes a framework for resource management in VPPs, which has been described as event-based because it utilizes the concepts of events and corresponding event processing derived from the resource classification and modeling to be introduced below. In the proposed framework, resources relevant to energy-management are classified into three tiers as shown in Figure 1:

- Domain Specific Resources (DSRs): These resources refer to those from different domains that contribute to the monitoring and control of energy generation/consumption. For instance, the information resource from the weather domain helps to define the forecast criteria for renewable energy generation, which in turn could alter the demand of energy from the main grid.

- Producer/Consumer Resources (PCRs): These resources refer to actors of the local power system such as customer-side generation units and controllable loads. For resources that may both consume and produce energy, such as a charging/discharging plugged-in electric vehicle (EV), they can be referred to as "prosumer" resources in this tier.

- Offered Energy Resources (OERs): These resources refer to customer-side offerings to supply, store, offload, or modify the demand of energy. For example, if a building with on-site generation (prosumer resource) generates more energy than it consumes, it may present to the VPP an offering to sell its surplus energy in the electricity spot market [3]. Such an offering is termed an offered energy resource (OER) in this tier. Furthermore, offerings from multiple PCRs, either co-located or geographically distributed but under the management of the same owner, can be aggregated and presented to the decentralized VPP as a single OER. We refer to an owner of PCR who participates in the VPP's intelligent energy planning/scheduling as an OER provider. The management of OERs is based on data aggregation, data analysis, and forecasting performed on PCRs. In this tier, the resource operations are typically market driven and DDM based.

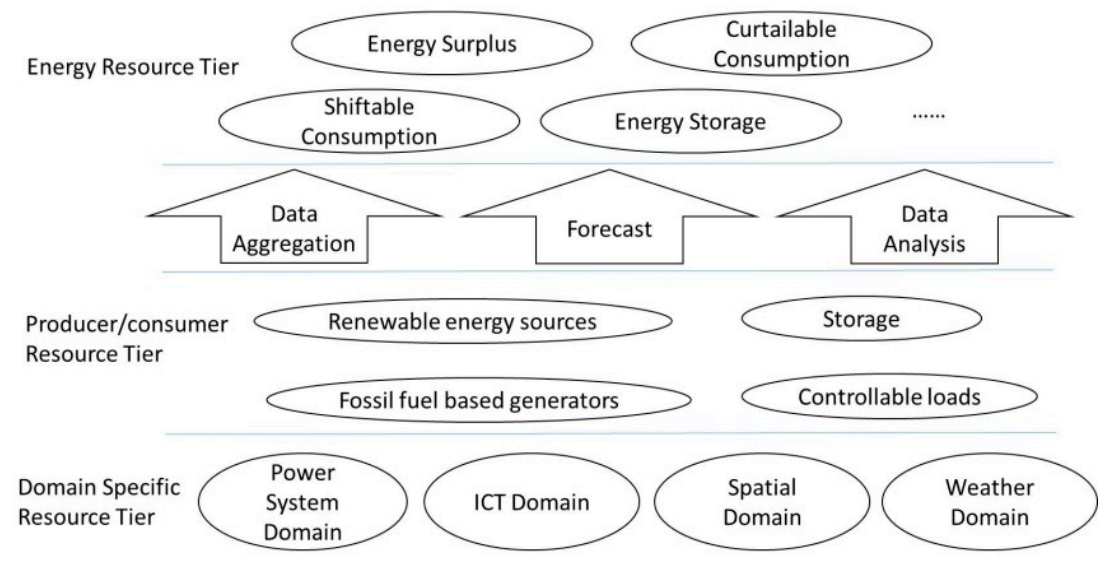

Figure 1. Framework for VPP Resource Management. 


\subsection{Primary Domains for Smart Grid}

There exist different domains of resources in VPP. Herein we identify the primary ones:

- Power System Domain: This is the domain that provides direct information about the PCR's energy consumption/generation profiles. In addition to what has been defined in the IEC suites, this domain should include operator-defined operating parameters (e.g., energy usage priorities) which can be used as criteria for energy scheduling and optimization.

- ICT Domain: This domain represents the information and communication technologies (ICT) that enable the smart operations of the electrical power grid. This includes smart objects such as networked sensors and actuators for automated facility monitoring and control. Each PCR may have one or more smart objects streaming data to or receiving control signals from the energy management systems over an information backhaul. Besides sensors and actuators, computing platforms, network infrastructure and data storage devices are some other ICT resources that are crucial to supporting smart energy management.

- Spatial Domain: Different spatial configurations of the building and spatial-use patterns of spaces within a building may lead to different consumption patterns of consumer resources inside them. Therefore, in addition to sensory data, spatial-related information of the buildings or facilities is an important resource to achieving accurate energy analysis.

- Weather Domain: Similar to spatial information, weather information also contributes to the analysis not only of energy consumption of buildings or facilities, but also of energy generation of on-site DERs. Resources in this domain can be shared among weather-dependent PCRs within the same locality, such as by streaming data from a local weather station to all buildings within the area to facilitate their consumption planning.

\subsection{Offered Energy Resources (OERs) Modeling and Problem Formulation}

The resource management problem in decentralized VPP where time is divided into consecutive time units, and real-time or forecast price signal is broadcast to each PCR is investigated. For example, the electricity wholesale market regulation in New Zealand defines each trading period as half an hour, for which a real-time price signal is broadcast [20].

The OER in VPP can be a contracted load curtailment volume from a consumer resource, or energy supplied from storage discharging on request. All OER providers in a decentralized VPP should be able to determine the optimal operation strategy based on forecast prices. If no dynamics are introduced, the VPP will be in an equilibrium condition where all participants have no intention to modify their consumption/generation patterns. However, a VPP in the Smart Grid is expected to react to energy consumption/generation changes. Those changes could be either a demand response request by the main grid to the VPP to curtail its overall energy demand for peak shaving, or a surge in energy demand experienced by an OER provider due to charging by a large number of visiting EVs unexpectedly. The VPP can select and aggregate one or more OERs in response to various energy change events originating from either within VPP or from the main grid. The definitions to formulate the above problem are as follows:

Definition 1: An energy state matrix (SM) is a matrix representing the energy state of a PCR or OER provider (with one or more PCRs). The matrix contains column-wise tuples of time slot index, volume of energy generated/consumed by PCR or energy exchanged between OER provider and VPP, and the corresponding revenue gain/loss over that time slot period. The revenue in SM is calculated based on electricity price and operational expenditure.

The following shows an example SM of a PCR (e.g., a building rooftop solar panel SP1) that contains hourly forecast values for a day (from time slot $T$ to $T+23$ ) where the values in first, second, and third row, represents the time in hourly slots, energy generated in $\mathrm{kWh}$, and revenue in cents/kWh, respectively: 


$$
\mathrm{SM}_{S P 1}=\left[\begin{array}{ccccccc}
T & T+1 & \ldots & T+7 & T+8 & \ldots & T+23 \\
0 & 0 & \ldots & 11.2 & 18.5 & \ldots & 0 \\
0 & 0 & \ldots & 10.7 & 10.7 & \ldots & 0
\end{array}\right]
$$

The energy and revenue values of the above SM at time slot $t=\{T, T+1, . ., T+23\}$ can be represented by matrix elements $\mathrm{SM}_{S P 1}(2, t)$, and $\mathrm{SM}_{S P 1}(3, t)$, respectively. Similarly, for a $\mathrm{SM}$ of an OER provider, the energy exchanged between an OER provider $i$ and VPP at time slot $t$, and the corresponding revenue, can be represented by $\mathrm{SM}_{i}(2, t)$, and $\mathrm{SM}_{i}(3, t)$, respectively.

Definition 2: An OER can be described by a parameter group composed of: (i) available time slot (TS); (ii) available energy volume $(Q)$; (iii) economic cost $(E c o C)$ incurred by revenue gain/loss; (iv) environmental $\operatorname{cost}(E n v C)$ incurred by environment deterioration during the resource's operation; and $(\mathrm{v})$ social welfare cost $(S w C)$ incurred by effects of resource's operation on power system social welfare. Therefore, a given OER $r$ can be represented as an array $\langle T S, Q, E c o C, E n v C, S w C>$ whose cost is given by a vector $C_{\text {vector }}$ :

$$
C_{\text {vector }}(r)=[\operatorname{EcoC}(r), \operatorname{EnvC}(r), \operatorname{SwC}(r)]
$$

Let $Q(r, t)$ denotes the energy volume provided by OER $r$, and $P^{*}(t)$ denotes the forecast prices for purchasing energy in future time slot $t$. The following presents the economic cost models for two different types of OERs:

\section{Type 1 OER: Curtailable consumption and dispatchable generation}

Curtailable consumption and dispatchable generation are offered from the OER provider to VPP as a modified amount of energy exchange from previous import planning (for consumption) or export planning (for generation) in a specified time slot only, i.e., there are no changes to the planning in other time slots. We group these two types of resources as type 1 OER.

Let the sign of the energy exchanged represents its flow direction: negative for importing energy while positive for exporting. For curtailable consumption, the OER provider reduces its energy consumption by an amount $\Delta \mathrm{Q}(\Delta \mathrm{Q}>0)$ in one or more time slots, potentially sacrificing utility (benefit) generated from energy consumption, e.g., occupant comfort, as a result. For dispatchable generation, the OER provider increases its energy production by an amount $\Delta \mathrm{Q}(\Delta \mathrm{Q}>0)$, potentially with more operational expenditure, e.g., by using more fuels. Figure 2 illustrates an example of type 1 OER. In Figure 2a, an OER provider has previously planned a consumption of Q1, and it offers a type 1 OER of reducing the consumption by $\Delta \mathrm{Q}$ to $\mathrm{Q} 2$. In (b), an OER provider has previously planned a generation of $\mathrm{Q} 1$, and it offers a type 1 OER of increasing the generation by $\Delta \mathrm{Q}$ to $\mathrm{Q} 2$.

As shown in Figure 2, these two OERs are similar in that they do not introduce inter-time slot dependencies, i.e., changing the energy planning by an amount $\Delta Q$ in time slot $t$ is only dependent on $P^{*}(t)$ and parameters of the aggregated PCR such as generation capacity. Thus, only column $t$ of their respective $\mathrm{SM}$ will be modified accordingly.

When scheduling such an OER $r$, the OER provider gains revenue from consumption reduction or generation increase based on $P^{*}(\mathrm{t})$, while losing revenue from sacrificial of utility or operational expenditure. Therefore, the economic cost for provisioning $\Delta \mathrm{Q}$ in time slot $t$ for type 1 OER can be given by:

$$
\operatorname{EcoC}_{\text {type } 1, r}(\Delta \mathrm{Q}, \mathrm{Q})=\left[P^{*}(\mathrm{t})-\lambda_{\text {type }, r}(\mathrm{t})\right] \times \Delta \mathrm{Q}
$$

where $\lambda_{\text {type1, } r}$ is the cost function of a type 1 OER $r$ to represent the utility sacrificed, or generation operational expenditure per energy unit. 


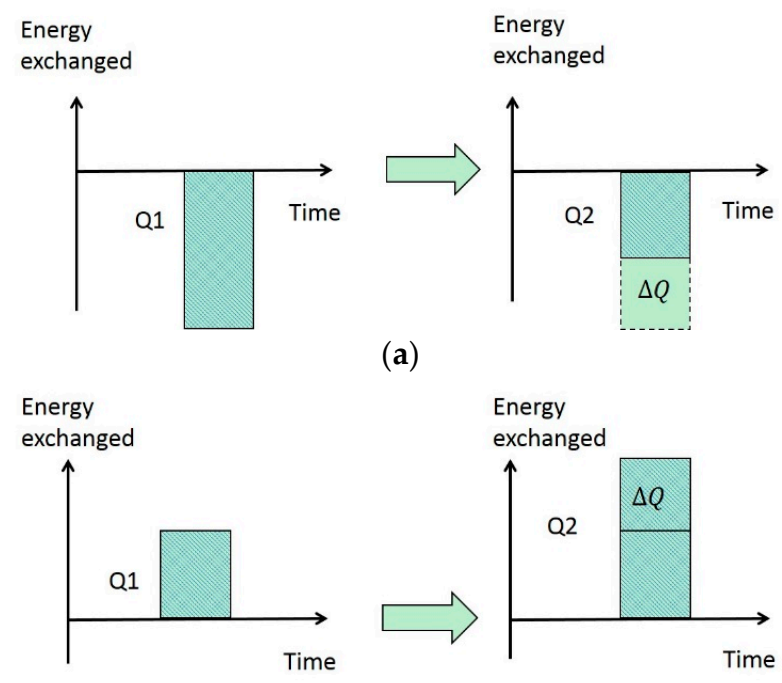

(b)

Figure 2. Illustration of type 1 OER: (a) Curtailable Consumption; (b) Dispatchable Generation.

\section{Type 2 OER: Shiftable consumption and storage charging/discharging:}

Shiftable consumption and storage charging/discharging are offered from the OER provider to VPP as a modified amount of energy exchange from previous import planning (for consumption and charging) and export planning (for discharging) in a specified time slot, with corresponding changes to the planning in one or more future time slots. These two types of resources are grouped as type 2 OER.

For shiftable consumption, the OER provider reduces (or increases) its energy consumption by an amount $\Delta \mathrm{Q}$ in time slot $t$, but increases (or reduces) its energy demand by the same amount in one or more future time slots. By shifting consumption to a different time slot, it potentially sacrifices some utility (benefit) that could have been generated from consumption in the original time slot $t$. For example, a cloud data center providing computation services may defer some of its clients' computation tasks (and thus the energy consumption associated with performing those tasks) to some future time slots, which in turn could sacrifice the delivered quality of services to its clients since it would take more time to complete their tasks. For energy storage systems, the OER provider charges to store (or discharges to provide) $\Delta \mathrm{Q}$ at time slot $t$, while discharges (or charges) by the same amount in one or more future time slots within a cycle time (typically a day). In addition to the electricity cost for charging, there are also costs associated with the operation and maintenance of the energy storage system, i.e., operational expenditure.

Figure 3 illustrates an example of type 2 OER. In Figure 3a, an OER provider reduces its consumption in the first time slot by an amount $\Delta \mathrm{Q}$, of which an amount $\mathrm{Q} 1$ and remaining amount Q2 is added to its consumption in second time slot, and third time slot, respectively. In Figure $3 b$, an OER provider charges its storage during the first time slot by an amount $\Delta Q$, of which an amount Q1 and remaining amount Q2 is discharged in second time slot, and third time slot, respectively.

When scheduling a shiftable consumption OER $r$, the OER provider gains revenue from consumption reduction in time slot $t$ based on $P^{*}(\mathrm{t})$, while potentially loses revenue from sacrificed utility and increased consumption in one or more future time slots. In the case of the storage system, the OER provider gains (loses) revenue from discharging (charging) energy in time slot $t$ based on $P^{*}(\mathrm{t})$, but loses (gains) revenue from charging (discharging) energy in one or more future time slot. Denoting $M$ and $\Delta Q_{j}$ as the set of one or more future time slots, and the amount of energy shifted to or charged/discharged in some future time slot $j \in M$, respectively, the economic cost for provisioning $\Delta \mathrm{Q}$ in time slot $t$ for type 2 OER can be given by:

$$
\operatorname{EcoC}_{\text {type } 2, r}(\Delta \mathrm{Q}, \mathrm{t}, \mathrm{M})=P^{*}(\mathrm{t}) \times \Delta \mathrm{Q}-\sum_{j \in M} \Delta Q_{j} \times\left[P^{*}(j)+\lambda_{\text {type } 2, r}(t, j)\right]
$$


where $\lambda_{\text {type2,r }}$ is the cost function of a type 2 OER $r$ to represent the utility sacrificed due to shifting consumption, or storage operational expenditure for charging/discharging per energy unit.
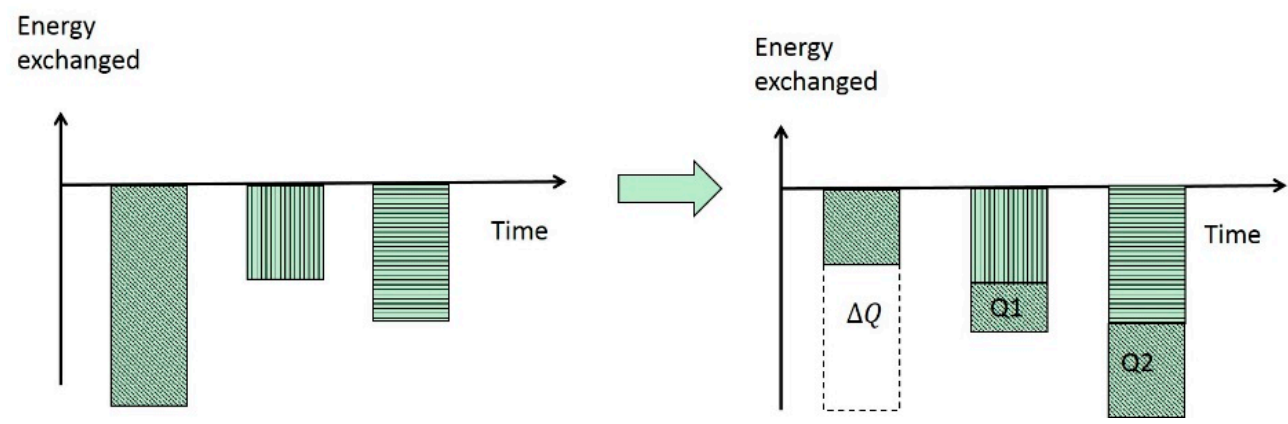

(a)

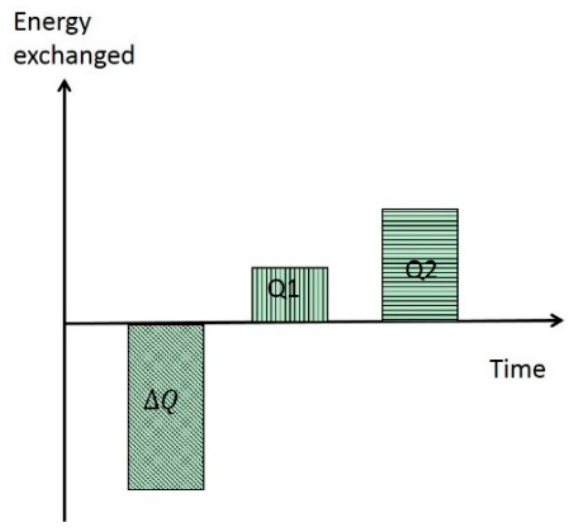

(b)

Figure 3. Illustration of type 2 OER: (a) Shiftable consumption; (b) Storage charging.

Since each OER provider has the forecast prices, for type 2 OER, the case where a future time slot set $\rho$ is proposed by the OER provider such that the economic cost incurred for every other possible time slot set is greater than the one in $\rho$, is investigated as follows:

$$
\rho=\underset{M}{\operatorname{argmin}} E c o C_{t y p e 2, r}(\Delta \mathrm{Q}, \mathrm{t}, \mathrm{M})
$$

To evaluate the social welfare of the power system, the day's peak average ratio (PAR) of the OER provider as the social welfare cost metric is utilized. Let $D$ represents the set of time slots for a day of operation. The social welfare $\operatorname{cost}(\mathrm{SwC})$ and the total $\operatorname{cost} C_{\text {total }}$ for OER $r$ from some OER provider $i$ can be given by Equations (5) and (6) respectively:

$$
\begin{gathered}
\operatorname{SwC}(\mathrm{r})=\operatorname{PAR}(\mathrm{r}, \mathrm{i})=\frac{\max _{t \in D}\left[\mathrm{SM}_{i}(2, t)+Q(r, t)\right]}{\underset{t \in D}{\operatorname{avg}}\left[\operatorname{SM}_{i}(2, t)+Q(r, t)\right]} \\
C_{\text {total }}(r)=\mathrm{f}\left(C_{\text {vector }}\right)=\mathrm{f}[\operatorname{EcoC}(r), \operatorname{Env} C(r), \operatorname{SwC}(r)]
\end{gathered}
$$

where $f()$ is the function agreed by VPP participants to evaluate the total cost of OER $r$ using cost metrics in $C_{\text {vector }}$. Furthermore, different resource scheduling algorithms that schedule resources according to VPP participants' cost/revenue, such as in [21,22] based on game-theoretic and genetic algorithmic approach, respectively, can be selected for application to the framework by substituting $f($ ) with the cost/revenue objective function of the applied algorithm.

With the above OER model, the problem is formulated as follows: given a set of time slots $t \in D$, available OERs $r \in R$, their cost metrics and an energy amount $G$ to be adjusted from previously 
planned energy consumption/generation, find a subset of OERs $\varphi \subseteq \mathrm{R}$, such that $\sum_{t \in \mathrm{D}} \sum_{r \in \varphi} Q(r, t)=G$, while $\sum_{r \in \varphi} C_{\text {total }}(r)$ is minimized.

\section{Events and Event Processing}

The resource selection for decentralized VPP is investigated as a DDM problem. The MAS approach is applied to implement one agent for each OER or PCR. The agent structure is shown in Figure 4. The event classification and processing of the proposed framework, in which the event sending/receiving between VPP participants is implemented based on the Foundation for Intelligent Physical Agents (FIPA) communication [23], are presented.

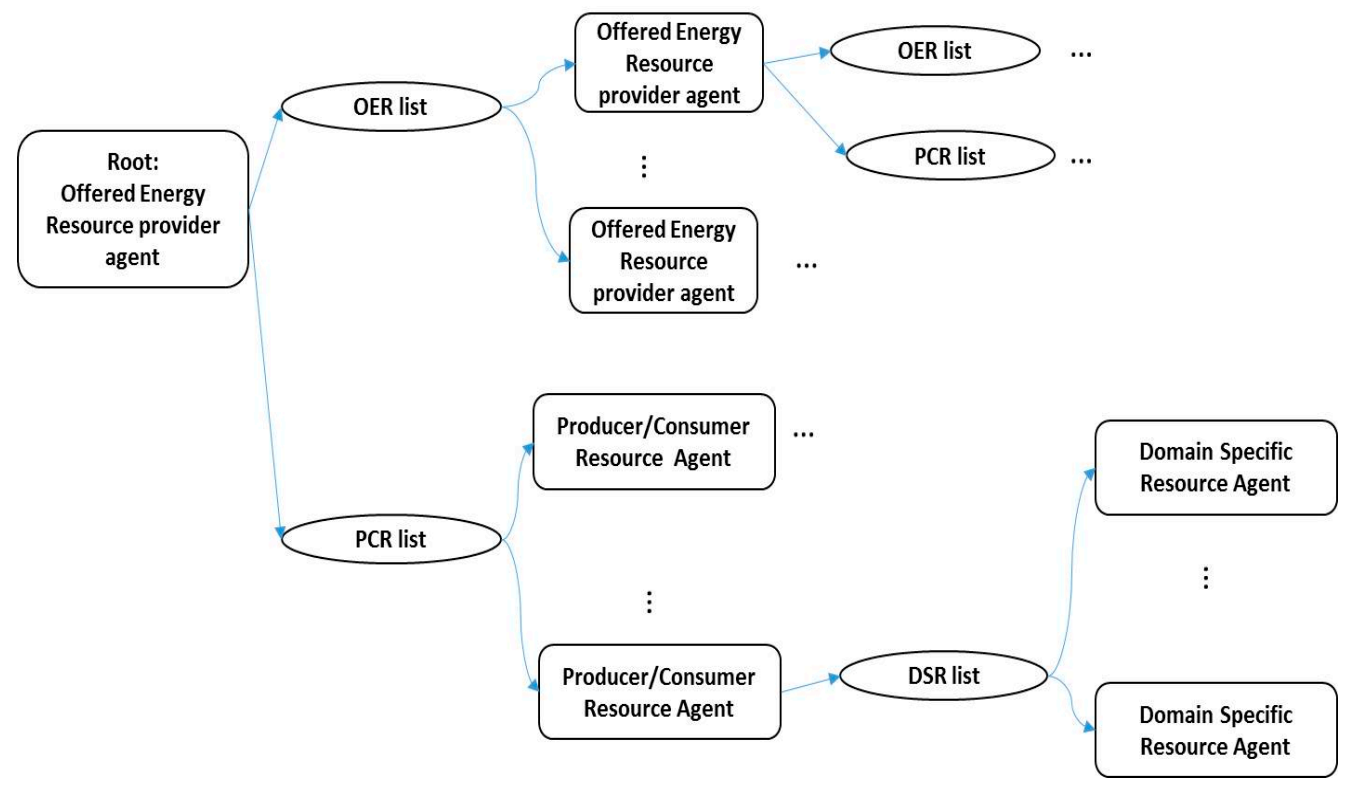

Figure 4. Agent Structure.

\subsection{Event Classification}

Based on the OER model, the event types are classified as follows:

- Energy Events: An energy event $e_{n}$ is triggered by a request for change in energy quantity over time. Typically, energy events are originated from an OER provider, and are sent/received between VPP participants in the energy resource tier of the proposed framework. An energy event can be described by a parameter group composed of: (i) request time slot (TS); (ii) change in energy volume $(\triangle Q)$; (iii) originated OER (OriOER); and (iv) next processing OER (NextOER). Therefore, a given energy event can be represented by an array $<T S, \triangle Q$, OriOER, NextOER $>$.

- Domain Events: A domain event $e_{d}$ can refer to a state transition event of a DSR or a PCR actuation event initiated by a DSR. Unlike energy events that are abstracted for resource selection, domain events are mostly discrete and occur as DSR state changes, or action tasks initiated by DSRs for PCRs such as the activation of an air-conditioner. They are a form of internal events communicated only within a VPP participant. Natural processes such as changes in the ambient temperature and solar radiation are considered as state transition events in this paper.

\subsection{Energy Event Routing}

An event routing algorithm for solving the DDM problem formulated in Section 3.3 is proposed. One provider agent represents each OER, and the VPP aggregation can be considered as a hierarchical tree with a root OER representing the overall VPP energy offerings to the main grid as shown in Figure 4 . In addition, the following definition is given: 
Definition 3: OERs and PCRs are siblings of each other if and only if they are directly aggregated by the same OER provider such as VPP operator or a customer owning several buildings. An OER is the parent (child) of another OER if it directly aggregates (is directly aggregated by) the other.

Each OER provider agent and PCR agent advertise their SMs to their sibling resources in order to calculate the social welfare cost metric, i.e., PAR, of the OER. Moreover, each OER provider agent maintains an energy event routing table (EERT) that dictates which OER is to be selected for an energy event, i.e., based on the information on this provider's OER, the sibling OERs, and a child OER with lowest cost among all children, the OER with lowest total cost can be identified by Equation (6). An example of EERT is shown in Figure 5 for OER_Customer_2.

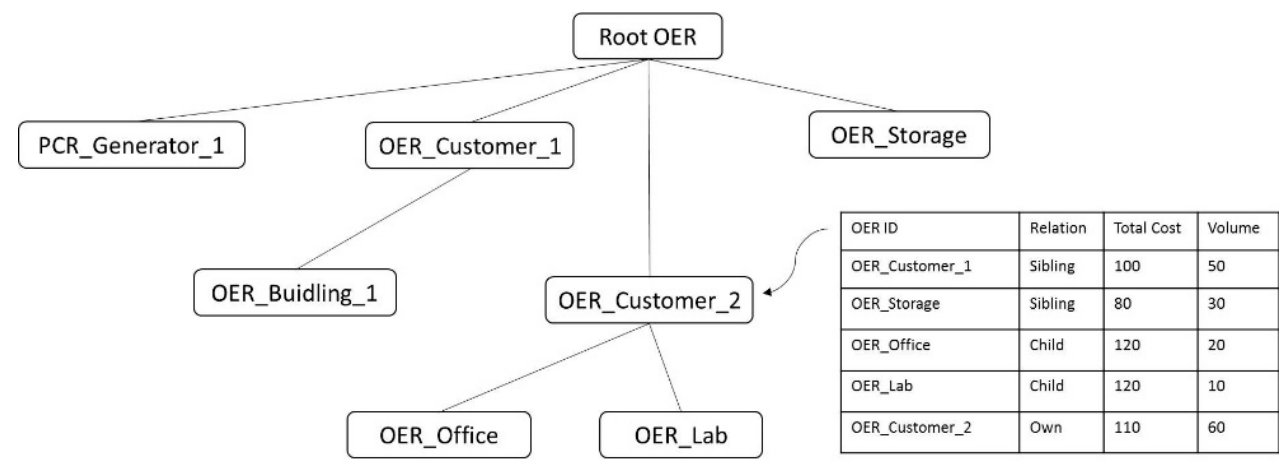

Figure 5. Example of VPP tree structure and energy event routing table.

The initialization algorithm for EERT (Algorithm 1) is shown in Figure 6. When an energy event is triggered, the OER provider agent that receives the event searches the EERT for the lowest cost OER.

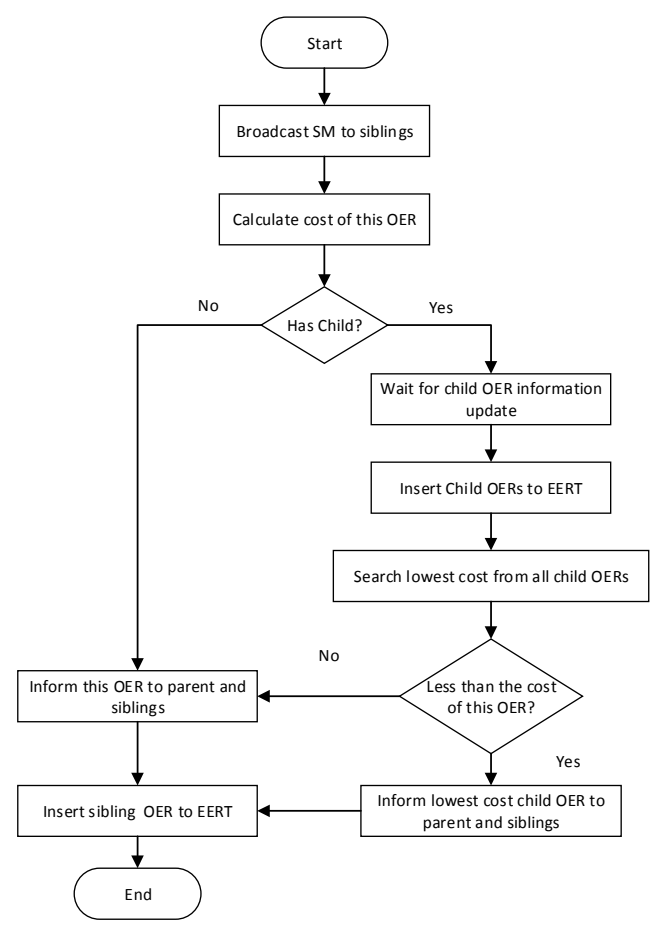

Figure 6. Energy Event Routing Table Initialization (Algorithm 1).

If the OER with lowest cost turns out to be its own OER, the provider agent performs the tasks required to provision the OER and propagates domain events to its PCR agents to control 
generation/consumption. If the lowest cost OER is one of its child OERs, the provider agent generates a new energy event with that child OER's available energy volume, sends the new event to that child and waits for its information update, informs sibling/parent OER on new cost if necessary before repeating the routing algorithm on the event with reduced energy volume. If the lowest cost OER is one of its sibling OERs, the provider agent sets the NextOER field of the event to that sibling OER and sends the event to its provider agent. The detailed algorithm (Algorithm 2) is shown in Figure 7.

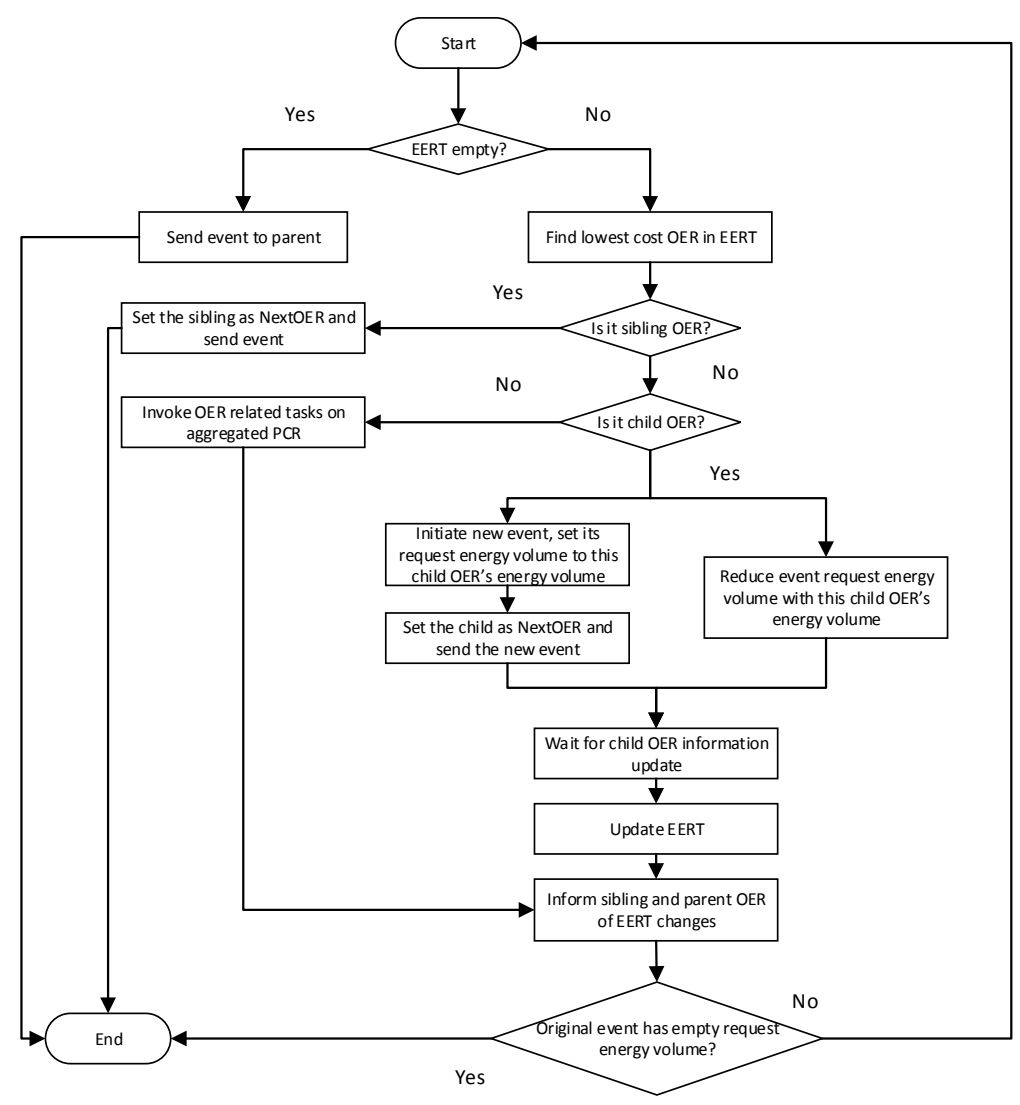

Figure 7. Energy Event Routing (Algorithm 2).

\section{Resource Management Framework Implementation}

Implementing the resource information modeling of the proposed framework requires addressing a number of essential issues:

- Shielding the Heterogeneity: The Smart Grid has been developed based on a myriad of different technologies, systems and devices. Legacy systems, i.e., outdated but still in use resource management systems, are also a primary concern for the evolving new standards that are being developed [1]. It is important for the resource model implementation to consider the problem of shielding its users from explicit handling of such heterogeneity and the interoperability between these heterogeneous elements. In the context of a VPP that aggregates multiple buildings, heterogeneity could also be introduced by the disparate energy management systems that may exist within different buildings of the VPP.

- Merging Different Domain Knowledge Bases: Different domains have different knowledge base formats, which usually come in the form of different domain ontologies. In order to achieve semantic interoperability between various domain ontologies, the resource model implementation should consider merging them under a top-level or upper ontology [24] for cross-domain synthesis of the resources in Smart Grid. 
- Predicting User Response: Having the capability to predict the responses of the energy users in different situations is important for VPP operation. The resource model implementation should facilitate the extraction of user parameters required by machine learning techniques such as Dynamic Bayesian Networks (DBN) for response prediction.

To meet the above requirements, the OWL is used to define the ontology of the framework, and thus the resources are semantically profiled using machine-understandable OWL files based on the model. This approach ensures the compatibility with existing standards and domain knowledge bases, as well as not overlapping with the power system models already defined in IEC 61850 and CIM, but can be incorporated into their future harmonization. Another important aspect is that with the ontology defined in the OWL, resource provisioning and management can utilize domain knowledge in interchangeable format, consequently allowing seamless integration of different software components within the VPP. Figure 8 shows the ontology implementation of the proposed resource model:

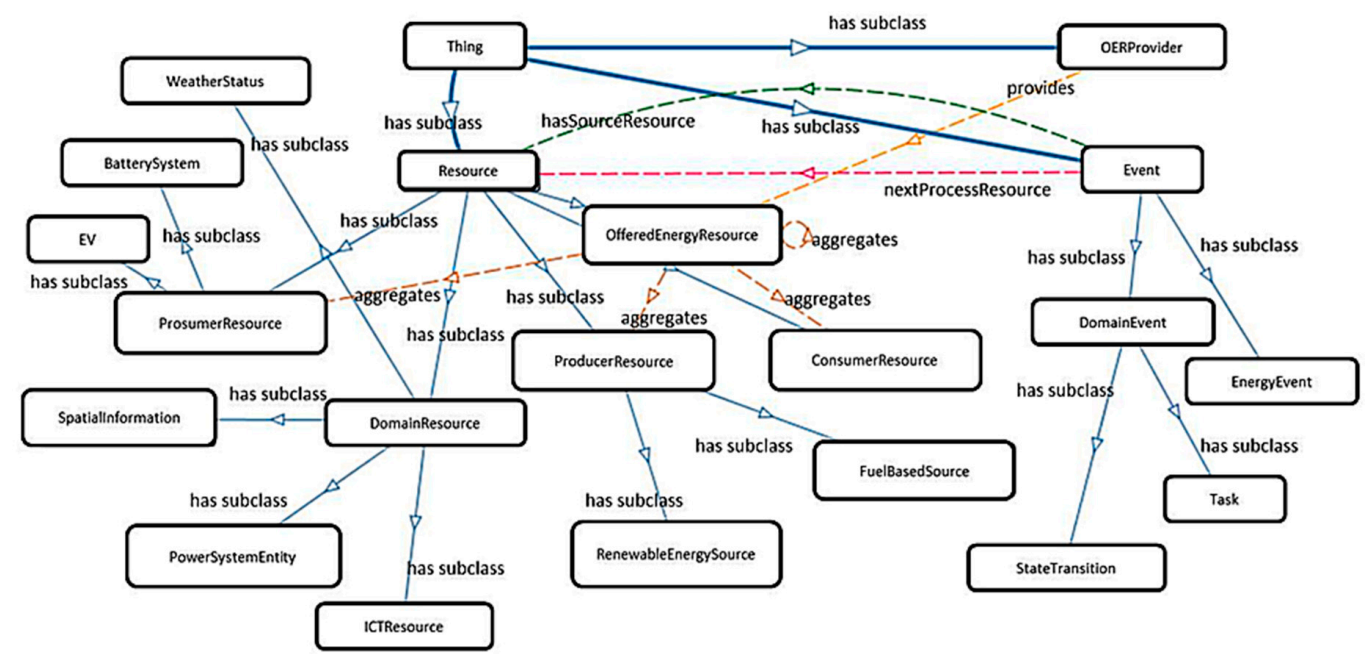

Figure 8. Ontology implementation.

A layered ontology structure is adopted, which is divided into upper ontology for OERs and PCRs, and domain ontology for DSRs. In the upper ontology, OERs are abstracted by the OfferedEnergyResource class, which has five types: LoadCurtailmentVolume, LoadShiftingVolume, EnergyConsumptionVolume, EnergyGenerationVolume, StorageChargeVolume and StorageDischargeVolume.

PCRs are abstracted by the ProducerResource, ConsumerResource, and ProsumerResource classes, which are designed to be the grouping points of different domain ontologies. Predicates such as dependsOnWeather are used to link the PCR with each domain class. New predicates could be introduced as the framework extends to encompass more domains.

In the domain ontology, DSRs are abstracted by the DomainResource class, whose subclasses include PowerSystemEntity, ICTResource, SpatialInformation, and WeatherStatus for power system domain, ICT domain, spatial domain, and weather domain, respectively. The PowerSystemEntity links to IEC/CIM harmonized device profiles and metering results by predicates hasProfile and hasMetering, whereas the SpatialInformation links to the resource's Building Information Modeling (BIM) knowledge base by predicate has SpatialInformation.

Events are classified into DomainEvent and EnergyEvent classes corresponding to previous event analysis. Event class has a predicate hasSourceResource to indicate its originating resource. The predicate nextProcessResource denotes the next receiver of event.

To extract knowledge from the resource information model, Simple Protocol and RDF Query Language (SPARQL) [25] is used to query the semantic database that stores the resource profiles. The SPARQL is a World Wide Web Consortium (W3C) standard query language for the semantic web. It can be easily integrated into enterprise software applications by using SPARQL engines such as Apache Jena [26]. 


\section{Results and Discussion}

This section evaluates the multi-agent system and ontology implementation of the proposed framework for a simulated campus VPP with real building energy data.

\subsection{Simulation Setup}

A full day energy scheduling of a decentralized VPP consisting of campus buildings, renewable generation, fuel cell and energy storage system is considered. Each building has an energy management system, which provides the energy consumption/generation interface to the MAS agents. The energy storage system is controlled by a prosumer resource agent. The topology of the simulated campus VPP is shown in Figure 9.

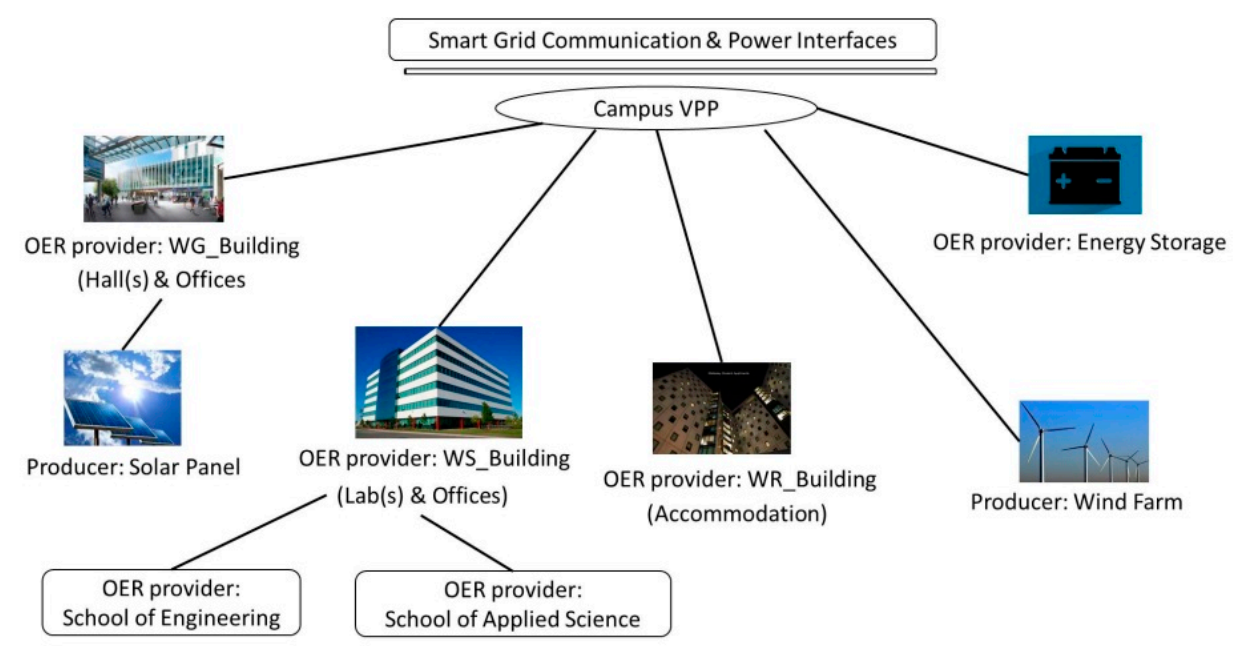

Figure 9. Simulated virtual power plant topology.

A normal workday (10 August 2015) during our winter semester is arbitrarily selected for the study case.

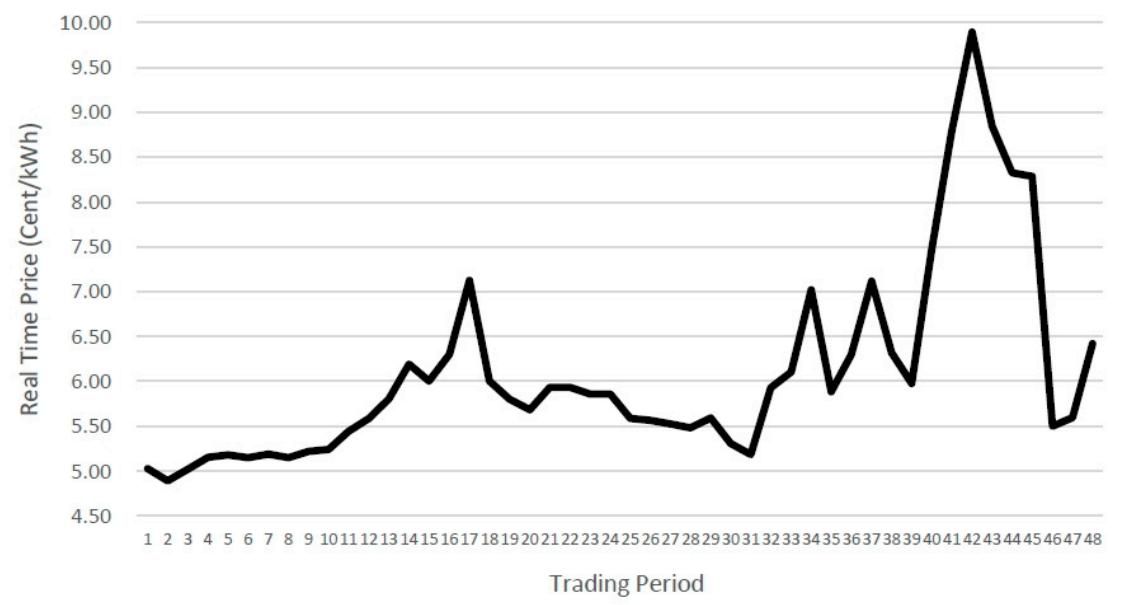

Figure 10. Electricity prices for the day 10 August 2015 from the Electricity Authority of New Zealand.

Figure 10 shows the half-hourly electricity price data for that day obtained from the Electricity Authority of New Zealand [27]. The price data is shown for 48 half-hourly trading periods over a full day of $24 \mathrm{~h}$. As demand forecasting is a research topic in itself which is beyond the scope of this paper, the historical metered energy data of Auckland University of Technology campus buildings are 
considered for the building energy consumption planning, i.e., future energy consumption pattern is considered to likely follow historical consumption pattern, which are shown in Figure 11. Without loss of generality, it is assumed that the energy storage system initially starts with a random value between zero and full storage capacity, and then subsequently (for next simulated days) starting with the end storage value from the previous day. The parameters of real world $500 \mathrm{kWh}$ storage systems [28] given in Table 1 are used for the simulated energy storage system. There is no environmental cost on OERs since the system only has renewable generation in VPP. The OERs of the buildings and their cost parameters are listed in Table 2.

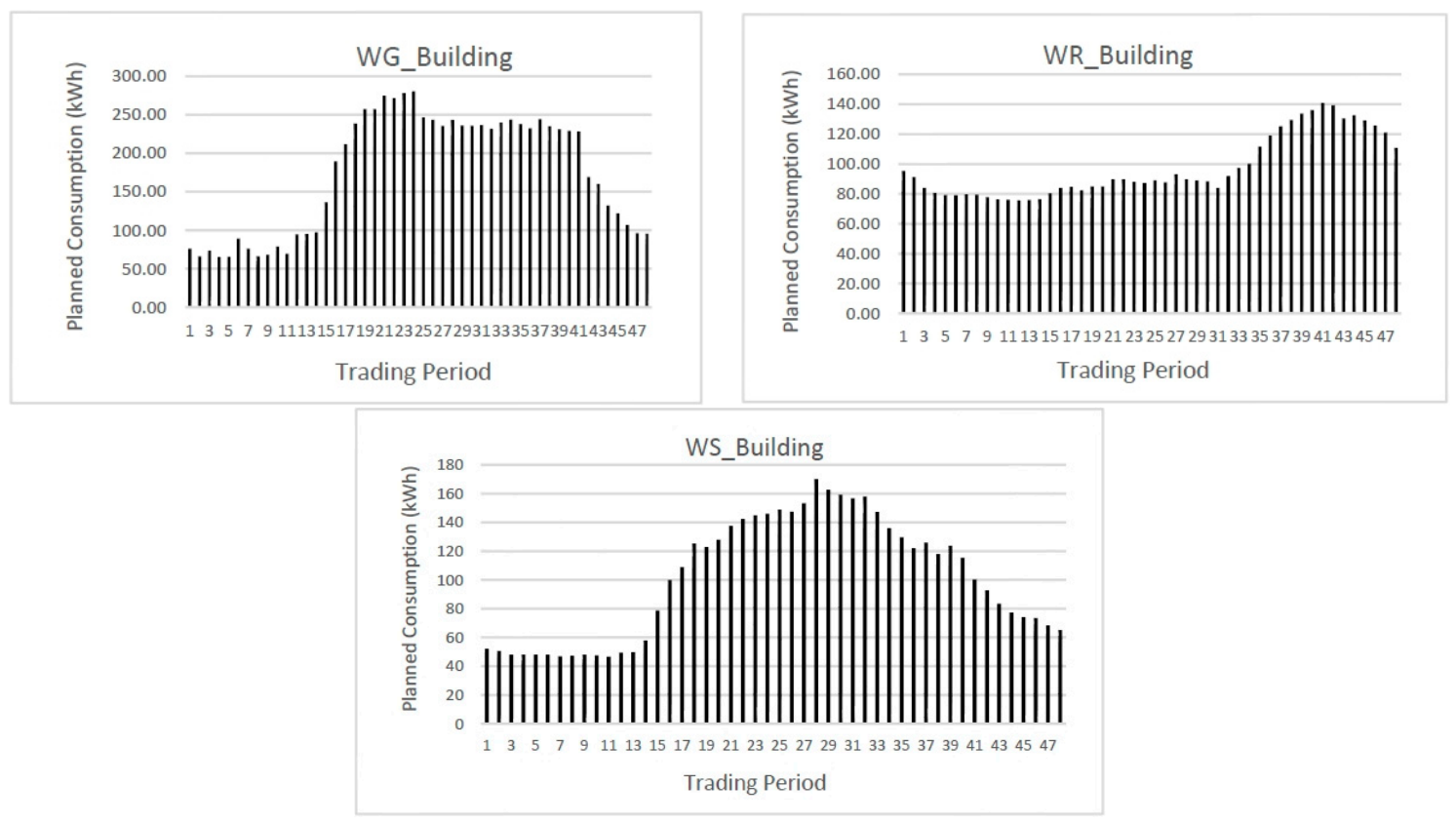

Figure 11. Building energy consumption planning from real-world historical data.

Table 1. Energy storage system parameters.

\begin{tabular}{cc}
\hline Storage Capacity & $\mathbf{5 0 0} \mathbf{~ k W h}$ \\
\hline Charge/discharge efficiency & $90 \%$ \\
Charge/discharge cost & 25 cents $/ \mathrm{kWh}$ \\
Maximum charge/discharge power & $250 \mathrm{~kW}$ \\
\hline
\end{tabular}

Table 2. OERs and cost parameters of campus virtual power plant (VPP).

\begin{tabular}{cccccc}
\hline Provider & ID & Volume & OER Type & Available TP & Cost Parameter \\
\hline WG_Building & G & $60 \mathrm{kWh}$ & 1 & $16 \sim 34$ & $\lambda_{\text {type } 1}=15$ \\
WR_Building & $\mathrm{R}$ & $20 \mathrm{kWh}$ & 2 & $16 \sim 40$ & $\lambda_{\text {type } 2}=10$ \\
WS_Building & $\mathrm{S}$ & $10 \mathrm{kWh}$ & 1 & $16 \sim 34$ & $\lambda_{\text {type } 1}=10$ \\
School of Engineering & S-E & $15 \mathrm{kWh}$ & 2 & $16 \sim 34$ & $\lambda_{\text {type2 }}=20$ \\
School of Applied & S-A & $15 \mathrm{kWh}$ & 2 & $16 \sim 34$ & $\lambda_{\text {type2 }}=18$ \\
Sciences & & & & &
\end{tabular}

The weather information used for renewable energy generation forecast is based on the weather profile for the same day obtained from New Zealand's National Institute of Water and Atmospheric Research (NIWA) [29]. The forecast solar and wind energy generation are shown in Figure 12. 

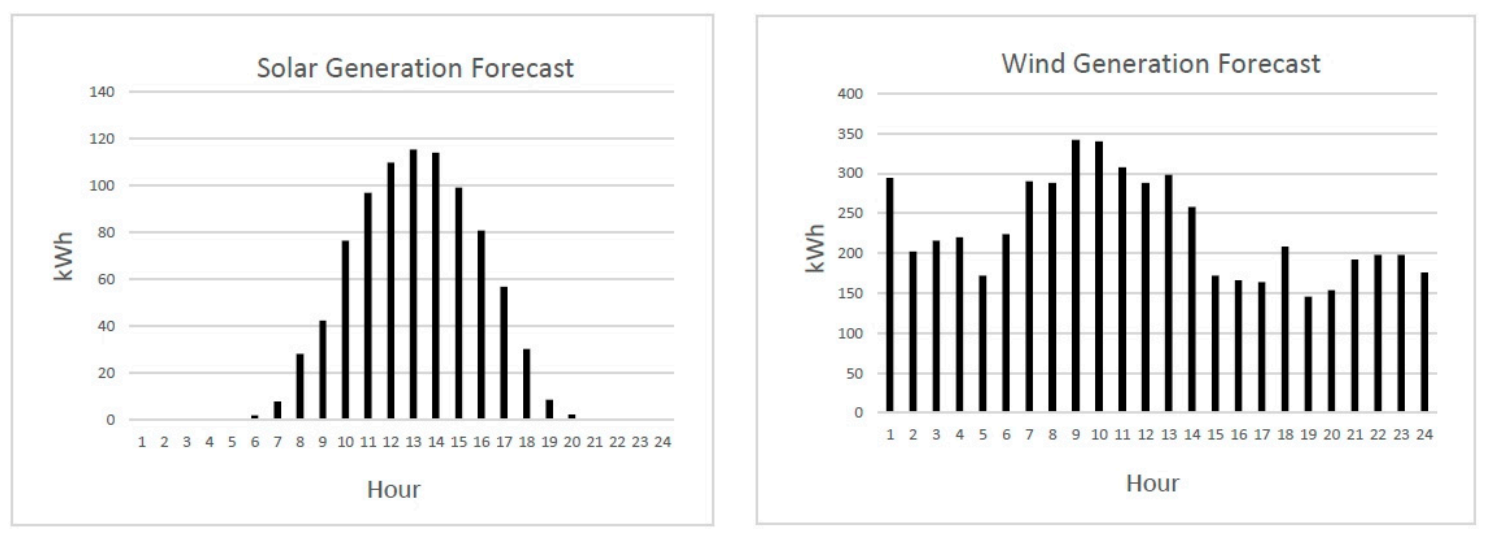

Figure 12. Renewable energy generation forecast.

Two types of energy events are introduced in the simulation: wind generation uncertainty and VPP generation plan. Since solar generation is generally more predictable than wind generation, the solar forecast generation is utilized as the actual solar generation. For wind generation, real-world data quality issues which affect its forecast accuracy are considered [30]. Thus, the higher-end value $(40 \%)$ of the forecast error range for wind generation is adopted. Therefore, at the beginning of each trading period (TP), an energy event will be triggered by the implemented MAS due to the observed difference between the forecast and actual wind generation. At TP 17, 34, 37 and 42 where electricity price peaks, the VPP will initiate an energy event to reduce demands and export as much energy as possible to the main grid.

\subsection{Simulation Results}

The simulated energy storage capacity for the day is shown in Figure 13, and the PAR of each OER provider and the whole VPP against their original planning is shown in Table 3. One can see that the energy storage is scheduled according to the price trends in Figure 10, and the PAR is reduced (closer to 1) compared to previously planned consumption, i.e., the VPP power system has increased the social welfare.

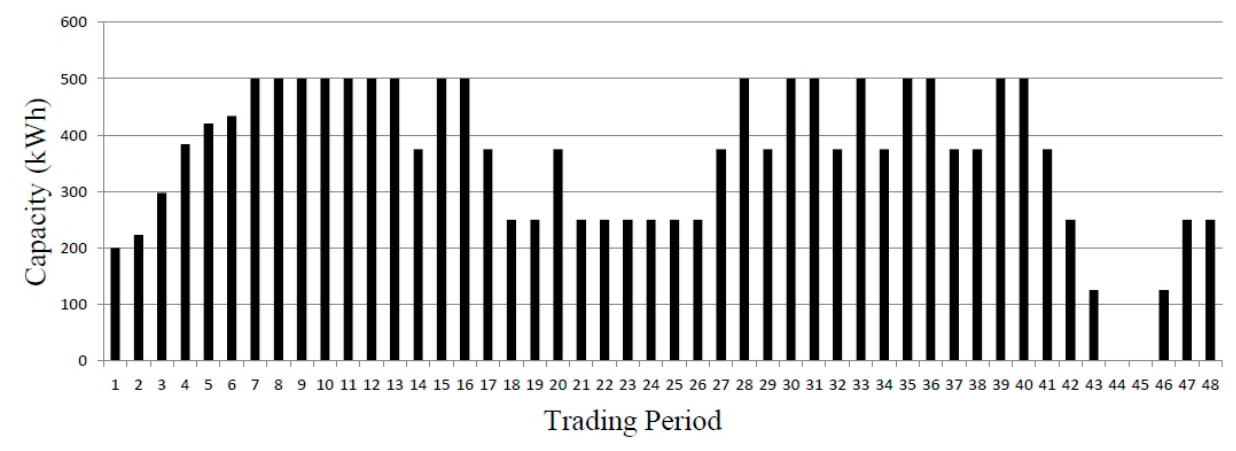

Figure 13. Energy storage system capacity.

Table 3. Peak-average ratio (PAR).

\begin{tabular}{ccc}
\hline Provider & PAR of Planned Consumption & Actual PAR \\
\hline WG_Building & 1.605 & 1.599 \\
WR_Building & 1.445 & 1.440 \\
WS_Building & 1.691 & 1.526 \\
Whole VPP & 1.378 & 1.337 \\
\hline
\end{tabular}


The investigation is further extended to larger VPPs with up to 100 buildings. The buildings are selected randomly as VPP members, with each building assigned to one of the three building types used in the previous simulation. For example, type $\mathrm{G}$ for halls/offices, type $\mathrm{S}$ for labs/offices, and type $\mathrm{R}$ for accommodation. Furthermore, the buildings have their energy consumption pattern normally distributed with real world consumption data as mean and 0.1 standard deviation. The cost/revenue objective function of the energy consumption game (ECG) [21] is utilized as the function $f()$ in Equation (6) for individual agents, along with a hybrid approach of using ECG for energy planning and our event-based resource selection for real time event processing.

The average results of the proposed event-based resource selection are compared with that of two other approaches: (a) without resource scheduling; and (b) real time ECG that runs the gradient algorithm for demand side management (DSM). In real time ECG, each agent iteratively sends out its energy planning according to price signals to coordinate with each other and adjusts consumption according to events. All agents run on the same computer with $3.2 \mathrm{GHz}$ i5 CPU with $8 \mathrm{~GB}$ memory, under 64-bit Windows 7 operating system. The result of VPP revenue, by net energy export of a day, is shown in Figure 14. The communication overhead (Figure 15) is also measured as the average number of messages sent from agents to one another before the scheduling result is finalized for a time slot. In addition, the computation overhead (see Figure 16) is measured as the average total computation time by all agents to finalize the scheduling result for a time slot, using Java Management Extension (JMX) profiling [31]. All results are shown with their $95 \%$ confidence intervals where applicable.

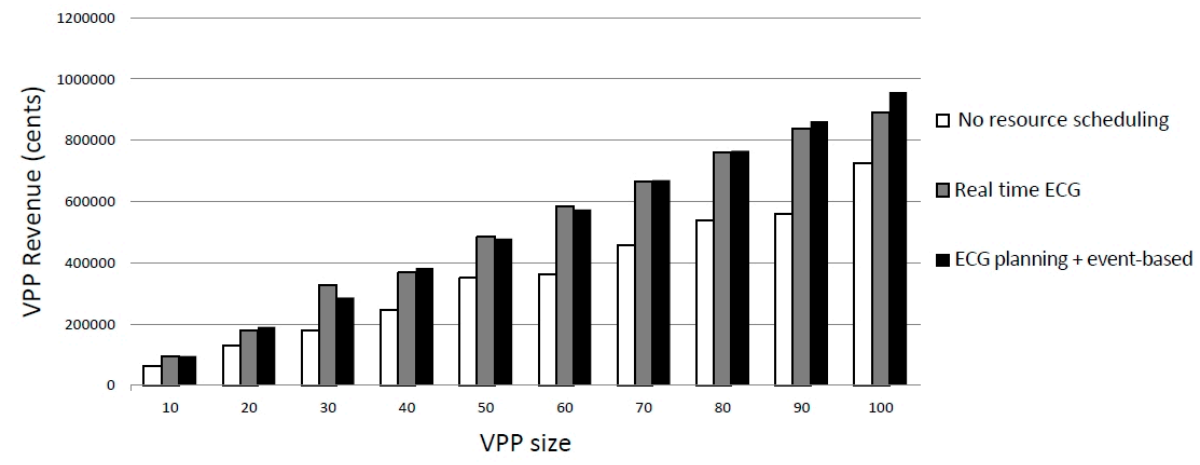

Figure 14. VPP Revenue Comparison.

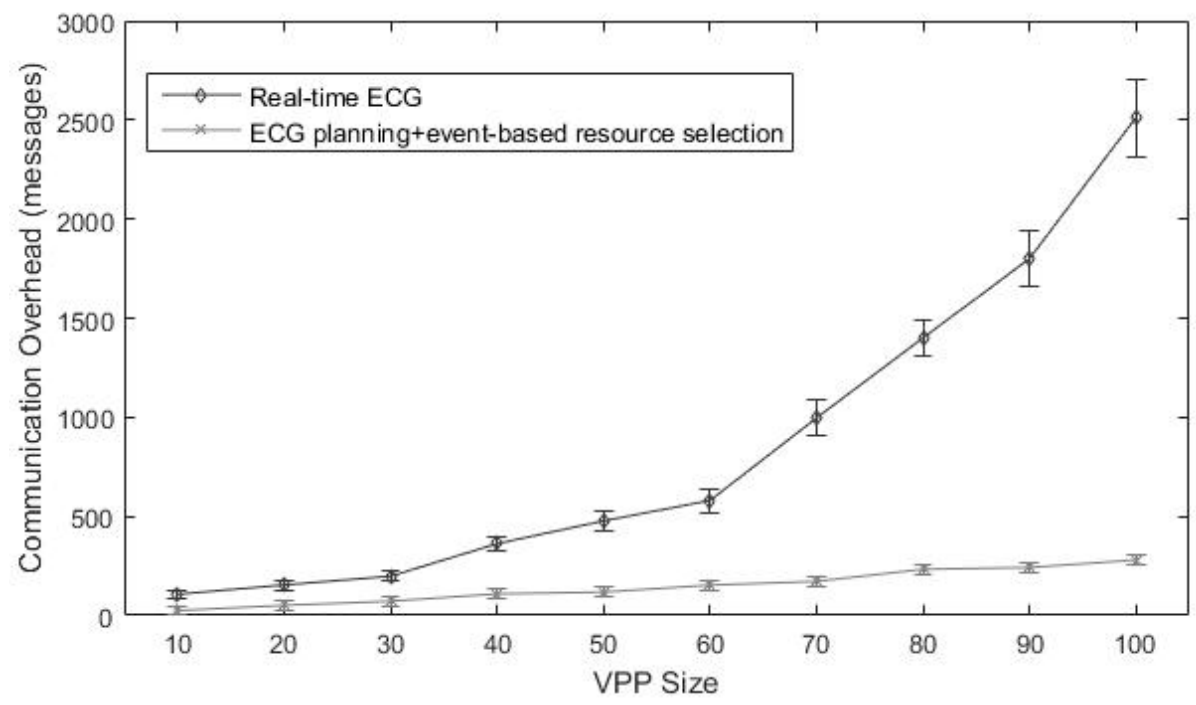

Figure 15. Communication Overhead Comparison. 


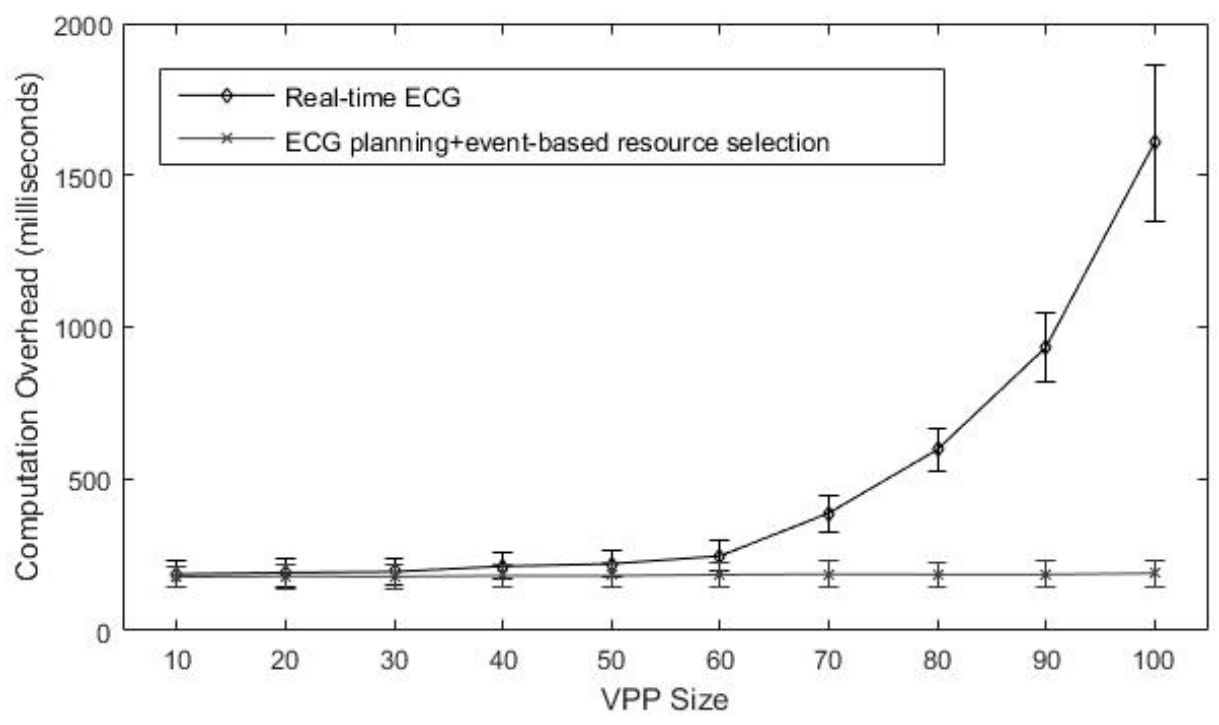

Figure 16. Computation Overhead Comparison.

The results show that both real time ECG and the approach based on the proposed framework expectedly performed better than without resource scheduling, and the improvement in revenue increases with the VPP size. It is also observed that the proposed approach achieves a revenue performance comparable with real time ECG, and performs better than real time ECG as VPP size increases to over 60 participants. Moreover, by not having to iteratively compute and send messages at every time slot, the proposed approach incurs significantly lower message and computation overheads as compared to real time ECG.

\section{Conclusions}

In Smart Grids, resource management is complex due to the wide variety of resources and the need for collaboration between systems in different domains. With a view to manage this complexity and to facilitate VPP application development, this paper proposes a framework, which views VPP as a hierarchical structure and abstracts consumption/generation from third party VPP participants for managing different resource types of relevance to energy-management in decentralized VPP.

Under the proposed framework, resource models and an event-based approach for distributed decision-making on resource selection are presented. The multi-agent system and ontology implementation of the framework are also presented. As evaluation, an analysis is conducted on a simulated campus VPP with real building energy data. The proposed approach has been shown not only to provide flexibility in making energy decisions in a distributed manner, but also to improve the overall revenue of the VPP with low communication and computation overheads. Therefore, the proposed framework could serve as a promising basis for future VPP automation design and accelerate development of cross-domain energy management applications for the Smart Grid.

Acknowledgments: The authors are grateful for the financial support provided by the Auckland University of Technology (AUT) for this research project. The authors would also like to thank Russell Baillie and Lucy McKenzie from the AUT Estates Office in facilitating our access to the energy data logs of the campus buildings.

Author Contributions: The topic and scope of this research were originally defined in a research proposal by Boon-Chong Seet and Tek Tjing Lie on smart powered buildings. Jianchao Zhang conducted the research and implemented the proposed solutions under the supervision of Boon-Chong Seet. The paper was drafted by Jianchao Zhang, and revised by Boon-Chong Seet and Tek Tjing Lie.

Conflicts of Interest: The authors declare no conflict of interest. 


\section{Abbreviations}

The following abbreviations are used in this manuscript:

$\begin{array}{ll}\text { BIM } & \text { building information modeling } \\ \text { CIM } & \text { common information model } \\ \text { CPS } & \text { cyber-physical system } \\ \text { CPU } & \text { central processing unit } \\ \text { DBN } & \text { dynamic bayesian network } \\ \text { DDM } & \text { distributed decision making } \\ \text { DER } & \text { distributed energy resource } \\ \text { DOLCE } & \text { descriptive ontology for linguistic and cognitive engineering } \\ \text { DSM } & \text { demand side management } \\ \text { DSR } & \text { domain specific resource } \\ \text { ECG } & \text { energy consumption game } \\ \text { ECoC } & \text { economic cost } \\ \text { EERT } & \text { energy event routing table } \\ \text { EnvC } & \text { environmental cost } \\ \text { FIPA } & \text { foundation for intelligent physical agents } \\ \text { ICT } & \text { information and communication technologies } \\ \text { IEC } & \text { international electrotechnical commission } \\ \text { IOT } & \text { internet of things } \\ \text { MAS } & \text { multi-agent system } \\ \text { OER } & \text { offered energy resource } \\ \text { OWL } & \text { ontology web language } \\ \text { PAR } & \text { peak average ratio } \\ \text { PCR } & \text { producer/consumer resource } \\ \text { JMX } & \text { java management extension } \\ \text { SLA } & \text { service level agreements } \\ \text { SM } & \text { state matrix } \\ \text { SSN } & \text { semantic sensor network } \\ \text { SwC } & \text { social welfare cost } \\ \text { SPARQL } & \text { simple protocol and RDF query language } \\ \text { TP } & \text { trading period } \\ \text { VPP } & \text { virtual power plant } \\ \text { W3C } & \text { world wide web consortium } \\ & \end{array}$

\section{References}

1. Joskow, P.L. Creating a Smarter US Electricity Grid. J. Econ. Perspect. 2012, 26, 29-47. [CrossRef]

2. Calderaro, V.; Galdi, V.; Lamberti, F.; Piccolo, A. A Smart Strategy for Voltage Control Ancillary Service in Distribution Networks. IEEE Trans. Power Syst. 2015, 30, 494-502. [CrossRef]

3. Bai, H.; Miao, S.; Ran, X.; Ye, C. Optimal Dispatch Strategy of a Virtual Power Plant Containing Battery Switch Stations in a Unified Electricity Market. Energies 2015, 8, 2268-2289. [CrossRef]

4. You, S.; Træholt, C.; Poulsen, B. A Market-based Virtual Power Plant. In Proceedings of the International Conference on Clean Electrical Power, Capri, Italy, 9-11 June 2009.

5. Schneeweiss, C. Distributed Decision Making-A Unified Approach. Eur. J. Oper. Res. 2003, 150, $237-252$. [CrossRef]

6. Naumann, A.; Bielchev, I.; Voropal, N.; Stycznski, Z. Smart Grid Automation Using IEC 61850 and CIM Standards. Control Eng. Pract. 2014, 25, 102-111. [CrossRef]

7. Liang, W.; Wang, N.; Wang, Z.; Liu, T.; Mu, Y. Research on Source Maintenance Key Technology of the Smart Substation. J. Power Energy Eng. 2014, 2, 239-243. [CrossRef]

8. Andrén, F.; Matthias, S.; Thomas, S. Towards a Semantic Driven Framework for Smart Grid Applications: Model-Driven Development using CIM, IEC 61850 and IEC 61499. Inform. Spektrum 2013, 36, 58-68. [CrossRef]

9. Santodomingo, R.; Rodríguez-Mondéjar, J.A.; Sanz-Bobi, M.A. Ontology Matching Approach to the Harmonization of CIM and IEC 61850 Standards. In Proceedings of the IEEE International Conference on Smart Grid Communications, Gaithersburg, MD, USA, 4-6 October 2010.

10. Benincasa, G.; D’Aniello, G.; Gaeta, M.; Loia, V.; Orciuoli, F. Resilient Semantic Sensor Middleware. In Intelligent Distributed Computing VIII; Springer International Publishing: Cham, Zug, Switzerland, 2015; pp. 453-463. 
11. Borgo, S.; Franssen, M.; Garbacz, P.; Kitamura, Y.; Mizoguchi, R.; Vermaas, P.E. Technical Artifacts: An Integrated Perspective. Appl. Ontol. 2014, 9, 217-235.

12. Goudarzi, H.; Pedram, M. Multi-Dimensional SLA-based Resource Allocation for Multi-Tier Cloud Computing Systems. In Proceedings of the IEEE International Conference on Cloud Computing (CLOUD), Washington, DC, USA, 4-9 July 2011.

13. Hu, G.; Zhu, W.; Niu, K. A User Model-Based Resource Scheduling Framework. In Proceedings of the IEEE International Conference on Green Computing and Communications (GreenCom), Beijing, China, 20-23 August 2013.

14. Salameh, K.; Chbeir, R.; Camblong, H.; Tekli, G.; Vechiu, I. A Generic Ontology-Based Information Model for Better Management of Microgrids. In Artificial Intelligence Applications and Innovations; Springer International Publishing: Cham, Zug, Switzerland, 2015; pp. 451-466.

15. Gillani, S.; Laforest, F.; Picard, G. A Generic Ontology for Prosumer-Oriented Smart Grid. In Proceedings of the 3rd Workshop on Energy Data Management, Athens, Greece, 28 March 2014.

16. Lopez, M.A.; de la Torre, S.; Martín, S.; Aguado, J.A. Demand-Side Management in Smart Grid Operation Considering Electric Vehicles Load Shifting and Vehicle-to-Grid Support. Int. J. Power Energy Syst. 2015, 64, 689-698. [CrossRef]

17. Karfopoulos, E.; Tenab, L.; Torresb, A.; Salasc, P.; Jordad, J.G.; Dimeasa, A.; Hatziargyrioua, N. A Multi-Agent System Providing Demand Response Services from Residential Consumers. Electr. Power Syst. Res. 2015, 120, 163-176. [CrossRef]

18. Hernandez, L.; Baladron, C.; Aguiar, J.M.; Carro, B.; Sanchez-Esguevillas, A.J.; Lloret, J.; Chinarro, D.; Gomez-Sanz, J.J.; Cook, D. A Multi-Agent System Architecture for Smart Grid Management and Forecasting of Energy Demand in Virtual Power Plants. IEEE Commun. Mag. 2013, 51, 106-113. [CrossRef]

19. Krauter, K.; Rajkumar, B.; Muthucumaru, M. A Taxonomy and Survey of Grid Resource Management Systems for Distributed Computing. Softw. Pract. Exp. 2002, 32, 135-164. [CrossRef]

20. NZX Limited. Wholesale and Information and Trading System (WITS) 30-Minute and 5-Minute Prices. Available online: http://www.electricityinfo.co.nz/comitFta/five_min_prices.main (accessed on 14 June 2016).

21. Su, W.; Huang, A.Q. A Game Theoretic Framework for a Next-Generation Retail Electricity Market with High Penetration of Distributed Residential Electricity Suppliers. Appl. Energy 2014, 119, 341-350. [CrossRef]

22. Silva, M.; Morais, H.; Vale, Z. An Integrated Approach for Distributed Energy Resource Short-Term Scheduling in Smart Grids Considering Realistic Power System Simulation. Energy Convers. Manag. 2012, 64, 273-288. [CrossRef]

23. O'Brien, P.D.; Nicol, R.C. FIPA—Towards a Standard for Software Agents. BT Technol. J. 1998, 16, 51-59. [CrossRef]

24. Chauhan, A.; Vijayakumar, V.; Ragala, R. Towards a Multi-level Upper Ontology/Foundation Ontology Framework as Background Knowledge for Ontology Matching Problem. Procedia Comput. Sci. 2015, 50, 631-634. [CrossRef]

25. Pérez, J.; Marcelo, A.; Claudio, C. Semantics and Complexity of SPARQL. ACM Trans. Database Syst. 2009, 34, 16:1-16:45. [CrossRef]

26. Apache Jena. Available online: http://jena.apache.org/ (accessed on 28 April 2016).

27. Electricity Authority of New Zealand. Electricity Market Information (EMI) Final Pricing Datasets. Available online: http://www.emi.ea.govt.nz/Datasets/Browse?directory=\%2FFinal_prices\& parentDirectory=\%2FDatasets\%2FWholesale\%2FFinal_pricing (accessed on 14 June 2016).

28. BYD Energy Storage Solutions. Available online: http://www.byd.com/energy/ess.html (accessed on 28 April 2016).

29. National Institute of Water and Atmospheric (NIWA). Available online: http://www.niwa.co.nz/ (accessed on 28 April 2016).

30. Huang, J.; Shiu, H.; van Dam, C.P. Wind Energy Forecasting: A Review of State-of-the-Art and Recommendations for Better Forecasts; California Institute for Energy and Environment: Berkeley, CA, USA, 2010.

31. ORACLE. Java SE Documentation, Chapter 2: Monitoring and Management Using JMX Technology. Available online: http://docs.oracle.com/javase/7/docs/technotes/guides/management/agent.html (accessed on 28 April 2016).

(c) 2016 by the authors; licensee MDPI, Basel, Switzerland. This article is an open access article distributed under the terms and conditions of the Creative Commons Attribution (CC-BY) license (http://creativecommons.org/licenses/by/4.0/). 